\title{
INFECÇÕES NO PÓS-TRANSPLANTE HEPÁTICO NA UNIDADE DE TRANSPLANTE HEPÁTICO DO HOSPITAL SANTA ISABEL
}

\author{
Infections in postoperative liver transplant of the Hospital Saint Isabel
}

\author{
Marcelo Scheidemantel Nogara, Ernani Tiaraju de Santa Helena, Cinthia Consolin Vieira, Lissandra Melati da Silva
}

\begin{abstract}
RESUMO
O transplante hepático pode ser considerado, sob o ponto de vista cirúrgico, uma das maiores intervenções já realizadas até hoje e tem como principais indicações a falência hepática aguda ou crônica por hepatite C ou B, ou cirrose alcoólica. Objetivos: Apresentar a incidência e distribuição topográfica das infecções hospitalares na Unidade de Transplante Hepático do Hospital Santa Isabel de Blumenau, no período de agosto/2002 a agosto/2006. Metodologia: Estudo coorte realizado através de análise retrospectiva dos prontuários de pacientes admitidos para transplante hepático até primeiro mês de pós-operatório, utilizando os critérios diagnósticos do Centro de Controle de Doenças dos Estados Unidos para as infecções encontradas. Resultados: Das 106 infecções diagnosticadas em 49 pacientes, os sítios de infecção mais freqüentes foram: infecções de vias aéreas inferiores (31\%) e infecções do sítio cirúrgico (23\%). Staphylococcus aureus foi isolado em 26,5\%, principalmente nas infecções de local cirúrgico e nas bacteremias. A principal etiologia de pneumonia foi a Klebsiella pneumonie. Conclusão: A incidência de infecção hospitalar foi de $61,25 \%$, sendo topografias mais comuns sítio cirúrgico e vias aéreas inferiores e o agente mais incidente foi o Staphylococcus aureus.
\end{abstract}

Descritores: Infecção, Período Pós-Operatório, Transplante Hepático.

\section{Instituição:}

Unidade de Transplante Renal do Hospital Universitário São José / Hospital Mater Dei Belo Horizonte / MG

Correspondência:

Euler Pace Lasmar

Rua Newtow, 89 - Belo Horizonte / MG - CEP: 30360-200 - Brasil

Tel.: (31) 32978848

E-mail: lasmar@superig.com.br

Recebido em: 16.06 .2008

\section{INTRODUÇÃO}

$\mathrm{O}$ transplante de fígado modificou a história natural de diversas doenças hepáticas e deve ser atualmente considerado uma realidade, tendo como principais indicações a falência hepática aguda ou crônica que ocorre principalmente por hepatite $\mathrm{C}$ ou $\mathrm{B}$, ou cirrose alcoólica. O primeiro transplante de fígado foi realizado com sucesso, em seres humanos, em 1963, por Thomas Starzl, em Denver, no Colorado. Desde então ocorreu um importante desenvolvimento na escolha e preservação dos órgãos doados, no refinamento da técnica cirúrgica, nos cuidados intra e pósoperatórios e nas medidas de imunossupressão visando o controle da rejeição. ${ }^{1-7}$

Desde o primeiro transplante hepático ortotópico realizado por Starzl, as infecções, nas suas mais variadas formas e etiologias, permanecem como uma constante e importante ameaça aos doentes candidatos ou já submetidos a esse tipo de tratamento. Embora a taxa de mortalidade relacionada às infecções dos pacientes pós-transplante hepático tenha diminuído substancialmente, a incidência de infecção após esse procedimento permanece elevada, sendo a principal causa de morte, principalmente no primeiro mês após a operação. ${ }^{1-7}$

\section{METODOLOGIA}

Pesquisa quantitativa, epidemiológica, aplicada, classificada como coorte. ${ }^{8}$ 
Para composição da amostra, foram analisados retrospectivamente os prontuário de todos os pacientes submetidos ao transplante hepático na Unidade de Transplante Hepático e suas complicações, no Hospital Santa Isabel, na cidade de Blumenau-SC, no período de agosto/2002 a agosto/2006. Todos os pacientes transplantados nesse período foram incluidos na pesquisa. Como critérios de exclusão, perda da amostra quando os pacientes eram transferidos para outras unidades hospitalares ou dados incompletos em prontuários que impossibilitavam a análise dos dados, foram elencados.

As variáveis envolvidas estiveram diretamente vinculadas à idade, sexo, indicação do transplante, tempo de internação, data do diagnóstico das infecções e o número de infecções por paciente.

O tempo de análise dos prontuários dos pacientes após o transplante hepático abrange o pós-operatório imediato até os primeiros 30 dias. O estabelecimento dessa contagem de dias foi baseado na subdivisão do Hospital Epidemiology and Infection Control da Filadelfia que didaticamente expõe três períodos de exposição à infecção em pacientes receptores de órgãos sólidos. Primeiro Período: compreende os primeiros trinta dias após o transplante, no qual ocorre a maioria das infecções e os fatores de risco estão relacionados ao procedimento cirúrgico e ao período de internação pós-operatório. ${ }^{9-10}$

Os critérios adotados para diagnóstico das infecções nosocomiais foram baseados no Center of Diseases Control and Prevention (CDC) que subdivide as infecções topograficamente em infecções de ferida cirúrgica classificadas em sítio superficial, profundo, de órgão ou cavidade (SSI); infecções do trato respiratório inferior (LRI); infecções primárias da corrente sanguínea (BSI); infecções do trato urinário (UTI); infecções do aparelho digestivo (AD); infecções oftalmológicas e infecções otorrinolaringológicas (EENT); infecções neurológicas (IN); infecções de pele ou partes moles (SST) e outras não enquadradas nas categorias anteriormente citadas. ${ }^{10-11}$ Os critérios foram rigorosamente seguidos, mesmo quando somente a característica clínica era evidenciada, não tendo obrigatoriedade de definições do agente etiológico.

A profilaxia antibiótica pré-operatória utilizada nesse serviço é tradicional o que gera igualdade em todos os pacientes avaliados. A cobertura antibiótica pré-transplante (dois inibidores das betalactamases e das penicilinas e um aminoglicosídeo) abrangendo germes gram positivos, negativos e germes anaeróbios, não prevenindo os atípicos. ${ }^{2}$ Pela Comissão de Controle de Infecção Hospitalar (CCIH) do Hospital Santa Isabel de Blumenau os agentes mais incidentes da unidade são o Staphylococcus aureus, Pseudomonas sp, Klebisiella pneumoniae, Enterococcus sp e o Acinetobacter baumanii.

A análise estatística foi através do Teste Exato de Fisher e T de Student. O estudo epidemiológico coorte evidenciou valores das taxas de incidência e riscos relativos. O cálculo de probabilidade usado foi baseado no método de Kaplan-Meier. ${ }^{8}$

\section{RESULTADOS DA PESQUISA}

Durante o período de agosto de 2002 a agosto de 2006 foram realizados, no Hospital Santa Isabel de Blumenau, 92 transplantes de fígado em pacientes adultos. Destes, 12 (13,4\%) foram excluídos.

Entre os 79 pacientes avaliados (um retransplante), 60 (75\%) eram do sexo masculino. As principais indicações dos 80 transplantes foram hepatite C (26\%) e cirrose alcoólica (17\%). A incidencia de infecções foi de $61,25 \%$ (49 pacientes). As etiologias das infecções no pós-operatório foram diagnosticadas em 45 casos, com um total de 106 infecções em 49 pacientes, com média de de 2 infecções por paciente (21\% dos casos).

O número de infecções apresentou diferenças quando comparado o gênero dos pacientes $\left(x^{2}=0,01580\right)$, apesar das mulheres terem representado $25 \%$ dos casos de transplante, nessa amostra apresentaram risco relativo de $8 \%$ em adquirirem infecções no pós-transplante hepático.

Quando calculadas as chances de um paciente transplantado adquirir uma infecção no pós-operatório através de curva de Kaplan-Maier, essa probabilidade chegou a $63 \%$ ao final do período avaliado, porém, mantendo oscilações significativas a partir do décimo quinto dia de internação.8

A Figura 1 evidencia os principais sítios de infecção quantificados na amostra estudada. Nele, as infecções de vias aéreas inferiores foram as mais incidentes (31\%), seguida das infecções de local cirúrgico (23\%).

Figura 1. Classificação e incidência de infecções topográficas.

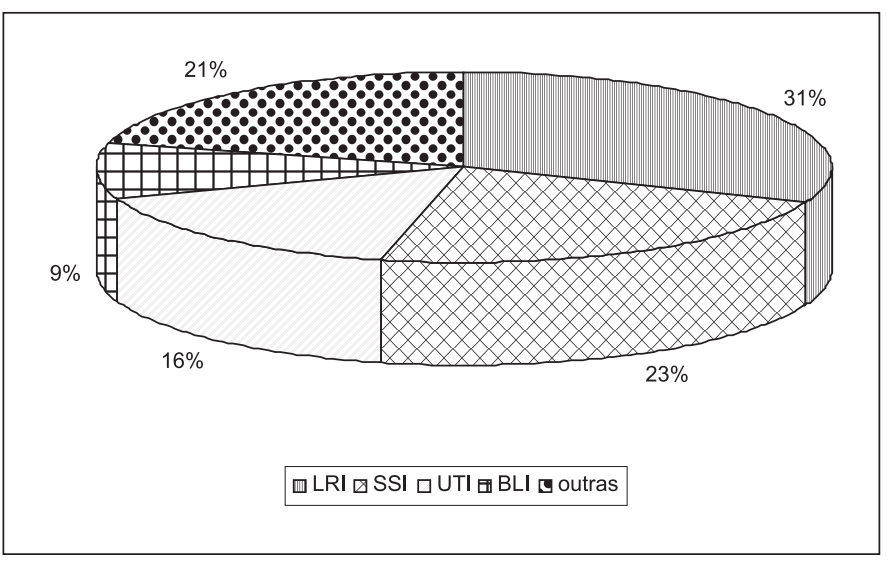

Identificou-se o agente em 45 das 106 infecções diagnosticadas. Trinta e oito foram causadas por bactérias, seis por fungos e três por vírus. Em quatro casos cresceram mais de um agente na cultura realizada. O Staphylococcus aureus foi o agente mais isolado das infecções bacterianas e a Candida albicans foi o fungo mais incidente (Figura 2).

Figura 2. Incidência das etiologias das infecções.

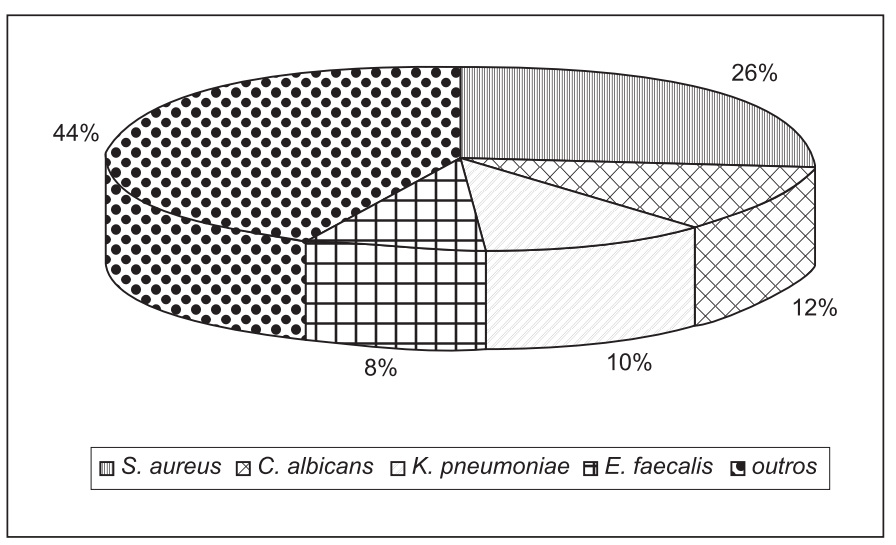


O S. aureus esteve presente em $16,7 \%$ dos casos diagnosticados de SSI e 28,6\% das BSI. A C. albicans foi responsável por todos os casos das EENT. O bacilo Gram negativo Escherichia coli foi encontrada em maior número nas UTI (50\%). A bactéria gram positiva Staphylococcus coagulase negativo foi a mais freqüente (25\%) das infecções de catéter venoso central.

Primeira causa de infecções no serviço, como topografia isolada, a pneumonia perfez 31 dos 32 casos das LRI. A bactéria gram negativa K.pneumoniae foi a mais freqüente dos sete casos onde o agente foi isolado, nos demais casos diagnosticados, o tratamento foi empírico.

A análise do índice de letalidade evidenciou uma taxa de 15\% nos trinta primeiros dias de pós-operatório, quando calculada a probabilidade de óbito pelo tempo de internação (Figura 3) essa alcançou $29 \%$ no trigésimo dia. BSI foi a única que se relacionou a um maior índice de óbitos (Teste de Fischer: $p=0,038$ ). Nos 10 casos de infecção de corrente, $40 \%$ dos pacientes foram a óbito. A taxa de letalidade relacionada às infecções de corrente sanguínea calculada foi de 0,33 .

Figura 3. Probabilidade de óbito por tempo de internação póstransplante.

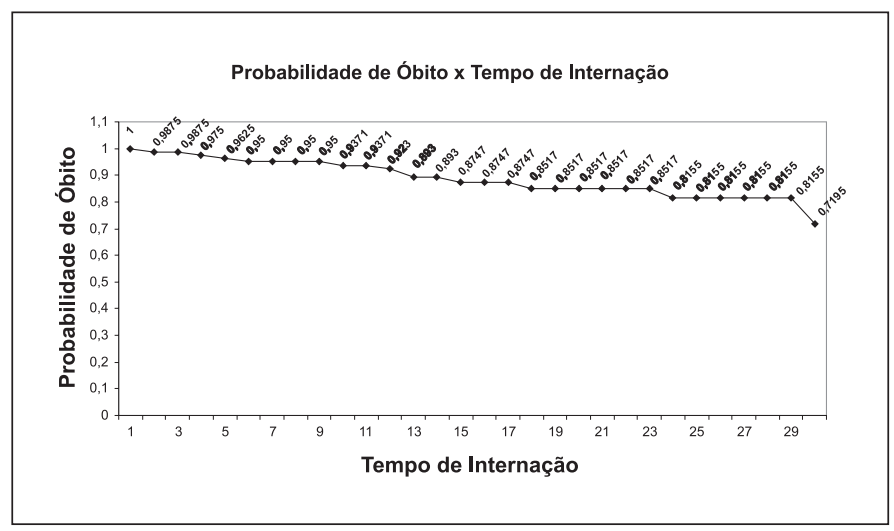

\section{DISCUSSÃO}

As principais indicações de transplante hepático caracterizando os receptores, em nossa casuística, foi hepatite $\mathrm{C}$ e cirrose alcoólica. Dados da literatura apontam essas indicações como relevantes, mas também incluem a hepatite criptogênica, vista em menores proporções nesse estudo (10\%). ${ }^{6,7,12-15}$

A taxa de infecções no pós-transplante nessa amostra foi de 60,75\%; na literatura comparada variam de 39 a $89 \% .^{6,7,12-14,16}$ As mulheres apresentaram risco relativo 8\% maior de apresentarem infecção quando comparada aos homens. A principal explicação estaria na afirmação de que a função hepática do receptor do órgão é inversamente proporcional ao número de infecções que ele pode adquirir no pós-operatório O sexo feminino foi responsável por indicações como hepatites auto-imunes ( 2 casos) e hepatites fulminante (2 casos). A hepatite fulminante é um diagnóstico que normalmente pressupõe um número aumentado de infecções, principalmente devido a gravidade dos pacientes, ao uso frequente de dispositivos invasivos, ao tempo prolongado de internação em terapia intensiva pré-transplante, à necessidade frequente de diálise e à própria insuficiência hepática. ${ }^{6,716}$
Em Freire e Coelho, $6,7,13,14$ as SSI representaram a maior parte das infecções, 35\% e 27,7\% respectivamente, diferentemente desse estudo, onde as LRI alcançaram 31\%, seguido das SSI com $23 \%$.

Quando comparado o agente etiológico, o S.aureus é descrito com um percentual de $20-25 \%$. A alta incidência se faz pela complexidade da intervenção cirúrgica e a alta colonização tanto pré como pós-transplante. Infecções por S.aureus são comuns em pacientes cirróticos, e esta, associada à insuficiência hepática. Há descrições de que esses pacientes tem diminuição na sua atividade imunológica, como quimiotaxia e fagocitose. .,7,13,14,16-19 $^{-1}$

As infecções fúngicas descritas foram exclusivamente por espécies de Candida, sendo esse agente responsável por 11,5\% das infecções gerais e $100 \%$ das causadas unicamente por fungos. O baixo uso de medicações anti-fungicas no pós-transplante pode explicar essa alta incidência, já que o uso de azólicos como o fluconazol não é muito indicado. As infecções por Candida são descritas entre 5 a $42 \%$, com tendência a queda nos últimos anos. ${ }^{6,7,13,14}$ Causas para maiores infecções por Candida estão relacionadas à diálise, retransplante, hepatite fulminante e uso de profilaxia para peritonite expontânea..$^{20,21}$

Alguns fatores de risco para infecção após o transplante hepático estão diretamente relacionados ao tempo de uso de dispositivos invasivos. Identificaram-se três dispositivos de uso invasivos mais comuns (sonda vesical, catéter venoso central e ventilação mecânica) como fator de risco para as infecções, apesar de normalmente os pacientes serem extubados até 12 horas após o transplante no Hospital Santa Isabel. Em nossa casuística a pneumonia foi a infecção mais diagnosticada, em um total de 31,12\%, dados da literatura oscilam entre $6 \%{ }^{16}$ e $20,3 \%,{ }^{11-13}$. As infecções do trato respiratório são a principal causa de morte por infecção hospitalar, sendo umas das infecções nosocomiais de difícil prevenção22. Num hospital não universitário do Rio Grande do Sul, a utilização da ventilação mecânica determinou um risco relativo de 3,44\% ao dia, para o desenvolvimento de pneumonia quando comparado ao risco de pacientes não ventilados. ${ }^{22}$

Manuais técnicos de prevenção a infecções hospitalares ${ }^{23,24}$ descrevem a disseminação comum pelo ambiente hospitalar de microorganismos que colonizam as superfícies horizontais dos mobiliários, como Staphylococcus aureus, Clostridium difficile, Pseudomonas sp, Proteus sp, Serratia mascescens e Candida sp.

De maneira influente sobre as SSI, a quantidade de sangue transfundida durante o transplante é um indicador indireto de volume de sangramento e, portanto, de cirurgia mais complicada, com maior manipulação, maior quantidade de tecido necrótico, menor concentração tecidual do antibiótico profilático e piores condições finais da ferida cirúrgica. Todos esses fatores contribuem com o risco de infecção. ${ }^{6,7,13,14,16}$

O diagnóstico de infecção primária de corrente sanguínea foi relativamente baixo no nosso estudo, representando apenas $9,43 \%$ (10 casos). Na revisão bibliográfica aqui apresentada, essa topografia teve variação de $12 \%$ a $30 \% .^{6,7,13,14,16} \mathrm{O}$ agente mais comumente encontrado nessa topografia foi o S. aureus, também não demonstrando relação com a literatura, ${ }^{6,7,13}$ que coloca o Acinetobacter baumanni e o Staphylococcus coagulase negativo como principais causadores de bacteremias. Fatores relacionados a uma maior incidência de BSI são o tempo de Unidade de Terapia Intensiva do doador, o uso de antibióticos pelo doador, idade 
do receptor, valor de creatinina do doador, tempo de ventilação mecânica e contagem de leucócitos do doador. ${ }^{7}$ Em média, 5\% dos doadores apresentam hemocultura positiva no momento do transplante, porém em algumas séries a positividade das hemoculturas chega a $23 \%{ }^{7}$

No que diz respeito probabilidade de óbito dentro dos 30 dias de pós-operatório, apresentada nesse estudo como $29 \%$, avaliouse como alta e apesar das descrições da literatura que elencam as melhorias da intervenção cirúrgica e cuidados no pré e pós operatório como grandes armas para o aumento da sobrevida esses percentuais ainda são preocupantes ${ }^{1,3}$. A topografia que mais se relacionou ao aumento da taxa de óbito, como esperado, foram as BSI, com taxa de mortalidade específica de $60 \%$. Freire et al identificaram uma transmissão de 3,7\% de doadores com culturas positivas e mortalidade em um ano de $45 \%$.

\section{CONCLUSÕES}

As infecções nosocomiais tem grande importância não só em relação a sua incidência, mas também por aumentar a morbi-mortalidade dos pacientes que são submetidos ao transplante hepático. As infecções encontradas na Unidade de Transplante Hepático do Hospital Isabel, apesar da alta incidência, estão relacionadas a topografias e locais comumente encontrados em outras unidades brasileiras.

É fundamental o controle rigoroso das infecções nosocomias pelo Centro de Controle de Infecções Hospitalares, através de um conjunto de medidas e cuidados que devem ser tomados pelos pacientes e funcionários da saúde: a limpeza, a esterilização e desinfecção correta dos materiais hospitalares bem como do ambiente hospitalar, para evitar a propagação e a disseminação dos microorganismos, em especial os multirresistentes, tendo em vista a busca incessante pela melhoria da qualidade da saúde.

\section{ABSTRACT}

Introduction: The liver transplant can be considered, under the surgical point of view, one of the biggest interventions that was developed until today and it has as main indications the acute or chronic liver bankruptcy caused by hepatitis C or B, or alcoholic cirrhosis. Purpose: To present the incidence and topographical distribution of the hospital infections in the Liver Transplant Unit of Santa Isabel Hospital from Blumenau, for a period from agosto/2002 to agosto/2006. Methods: this is a cohort study developed through retrospect analysis of handbooks from admitted patients for liver transplant until first month of postoperative, using the disgnostics criterions of the Control Center of Illness of the United States for the found infections. Results: 106 infections in 49 patients was identified. The localities of most frequent infections were: infection of lower airways (31\%) and surgycal wound infection (23\%). Staphylococcus aureus was the most isolated agent, with $26,5 \%$ of the infections, being more prevalent in surgycal wound and bacteremia infections. The main etiology of pneumonia was Klebsiella pneumonie. Conclusion: The incidence of hospital infection is $61,25 \%$. The most common topographies of infections are surgycal wound infection and lower airways infection. The most incident agent was Staphylococcus aureus.

Keywords: Infections, Postoperative, Liver transplant

\section{REFERÊNCIAS}

1. Dani R. Gastroenterologia essencial. 3. ed. Rio de Janeiro : Guanabara Koogan; 2000.

2. Cecil JC, PLUM F. Tratado de medicina interna. Tradutores Amaury José da Cruz Júnior ... et al. 21.ed. Rio de Janeiro : Guanabara Koogan; 2001.

3. Coelho J. Aparelho digestivo: clinica e cirurgia. 2.ed. Rio de Janeiro : MEDSI; 1996.

4. Cordeiro FTM. Condutas em gastroenterologia Rio de Janeiro : Revinter; 2004.

5. Lima LP. Condutas em cirurgia hepatobiliopancreatica. Rio de Janeiro: MEDSI; 1995.

6. Coelho J. Incidência de Infecções em Unidade de Transplante Hepático. Registro Brasileiro de Transplante. 2002,(3):3-20.

7. Freire MP. Infecções Hospitalares em pacientes submetidos a transplante de fígado: fatores de risco relacionados ao doador [dissertação]. Faculdade de Medicina do Estado de São Paulo; 2006.

8. Massad E, Menezes RX. Métodos Quantitativos em Medicina 1.ed. Barueri - SP: Manole; 2005.

9. Snydman DR. Epidemiology of infections after solid-organ transplantation. Clin Infect Dis. 2001;33(6): 5-8.

10. Associação Nacional de Vigilância Sanitária. Diagnóstico do Controle da Infecção Hospitalar no Brasil. 2005;(5):1-19.

11. Centers os Disease Control and Prevention. Case definitions for infectious conditions under public health surveillance. MMWR 1997.

12. Chang FY, Singh N, Gayowsky T. Fever in liver trasnplant recipients: changing spectrum of etiology agents. Clin Infect Dis. 1998;26(3):59-65.

13. Coelho JC, Parolin M, Matias JE. Causas de óbito tardio em transplantados de fígado. Revista da Associação Médica Brasileira. 2003;49(4-5):177-80.

14. Coelho JC, Parolin M, Matias JE. Infecções Hospitalares bacterianas em Unidade de Transplante Hepático. Jornal Brasileiro de Transplantes. 2004;7:198-202
15. Kruger M. European hepatitis B immune globulin trials: prevention of recurrent hepatitis B after transplantation. Clin Transplantation. 2000;14(2):14-9.

16. Losada I, Cuervas VM. Infection precoz em el paciente com transplante hepático: incidência, gravedad, factores de riesgo y sensibilidad antibiótica de los aislados bacterianos. Enferm Infecc Microbiol Clin. 2002;20(9):422-30.

17. Gouvea EF, Branco RC. Outcome of infections caused by multiple drug-resistant bactéria in liver transplant recipients. Transplantation Proceedings. 2004;36(4):958-60.

18. Singh N, Patterson DI. Methicilin-resistant Staphylococcus aureus: the other emerging resistant gram-positive coccus among liver transplant recipients. Clin Infect Dis. 2000;30(2):322-7.

19. Torre-Cisneros J, Herrero C. High mortality related with Staphylococcus aureus bacteremia after liver transplantation. Eur J Clin Microbiol Infect Dis. 2002;21(5):385-8

20. Singh N, Wagener MM, Marino IR. Trends in invasive fungal infections in liver transplant recipients: correlation with evolution in transplantation practices. Transplantation. 2002;73(1):63-7.

21. Husain S, Tollemar J. Changes in the spectrum and risk factors for invasive candidiasis in liver transplant recipients: prospective, multicenter, case-controlled study. Transplantation. 2003;75(12).

22. Silva NB, Ravanello ML, Cantarelli M. Pneumonia nosocomial em pacientes críticos. Análise dos resultados obtidos em UTI de adultos do Hospital de Moinhos de Vento durante cinco anos. Bisturi. 2003;135:26-8

23. Parolin MB, Zaina FE, Lopes R. Terapia Nutricional no Transplante Hepático. Arquivos de Gastroenterologia. 2002;39(2)

24. Andrade D, Angerami E, Padovani CR. Condição microbiológica dos leitos hospitalares antes e depois de sua limpeza. Revista de Saúde Pública. 2002;34(2). 